submitted to ApJ

\title{
The Lithium abundances of a large sample of red giants
}

\author{
Y.J. Liu, ${ }^{1}$, K. F. Tan $^{1}$, L. Wang ${ }^{1}$, G. Zhao, ${ }^{1}$, Bun'ei Sato, ${ }^{2}$, Y. Takeda ${ }^{3}$, H.N. Li, ${ }^{1}$
}

\begin{abstract}
The lithium abundances for $378 \mathrm{G} / \mathrm{K}$ giants are derived with non-LTE correction considered. Among these, there are 23 stars that host planetary systems. The lithium abundance is investigated, as a function of metallicity, effective temperature, and rotational velocity, as well as the impact of a giant planet on $\mathrm{G} / \mathrm{K}$ giants. The results show that the lithium abundance is a function of metallicity and effective temperature. The lithium abundance has no correlation with rotational velocity at vsini $<10 \mathrm{~km} \mathrm{~s}^{-1}$. Giants with planets present lower lithium abundance and slow rotational velocity $\left(\right.$ vsini $\left.<4 \mathrm{~km} \mathrm{~s}^{-1}\right)$. Our sample includes three Li-rich $\mathrm{G} / \mathrm{K}$ giants, 36 Li-normal stars and 339 Li-depleted stars. The fraction of Li-rich stars in this sample agrees with the general rate of less than $1 \%$ in literature, and the stars that show normal amounts of Li are supposed to possess the same abundance at the current interstellar medium. For the Lidepleted giants, Li deficiency may have already taken place at the main sequence stage for many intermediate-mass $\left(1.5-5 \mathrm{M}_{\odot}\right) \mathrm{G} / \mathrm{K}$ giants. Finally, we present the lithium abundance and kinematic parameters for an enlarged sample of 565 giants using a compilation of literature, and confirm that the lithium abundance is a function of metallicity and effective temperature. With the enlarged sample, we investigate the differences between the lithium abundance in thin-/thick-disk giants, which indicate that the lithium abundance in thick-disk giants is more depleted than that in thin-disk giants.
\end{abstract}

Subject headings: methods: data analysis - stars: abundances — stars: late type — techniques: spectroscopic

\footnotetext{
${ }^{1}$ Key Laboratory of Optical Astronomy, National Astronomical Observatories, Chinese Academy of Sciences, A20 Datun Road, Chaoyang District, Beijing 100012; lyj@nao.cas.cn, gzhao@nao.cas.cn

${ }^{2}$ Tokyo Institute of Technology, 2-12-1 Ookayama, Meguro-ku, Tokyo 152-8551, Japan

${ }^{3}$ National Astronomical Observatory of Japan, 2-21-1 Osawa, Mitaka, Tokyo 181-8588, Japan
} 


\section{Introduction}

Lithium is an important element in the understanding of chemical evolution history of the Galaxy, as well as in the study of the mixing process in stellar interiors. As is known, the lithium abundance is believed to be a function of metallicity, effective temperature, stellar mass, age, stellar rotation and chromospheric activity. The behavior of lithium as a function of effective temperature has been well studied both for subgiants (De Medeiros et al. 1997; Lèbre et al. 1999; Randich et al. 2000; Mallik et al. 2003) and giants (Brown et al. 1989; Wallerstein et al. 1994; De Medeiros et al. 2000; de Laverny et al. 2003, Lèbre et al. 2006). Based on these literatures, we can conclude that the abundance of lithium becomes depleted with decreasing effective temperature among stars with spectral types of $\mathrm{G}$ to $\mathrm{K}$, while also showing a wide dispersion for stars with spectral types of F8 to G0. Such behavior demonstrates that convective mixing is more severe for $\mathrm{G}$ and $\mathrm{K}$ type stars. Therefore, giants with spectral types of $\mathrm{G}$ and $\mathrm{K}$ are expected to show lower lithium abundances.

The lithium behavior as a function of stellar rotation (e.g. De Medeiros et al. 2000, de Laverny et al. 2003, Lèbre et al. 2006) and chromospheric activity (Ghezzi et al. 2010, Takeda et al. 2010) have also been studied by many investigations. It is suggested that fast rotation and active chromospheric activity lead to high $\mathrm{A}_{\mathrm{Li}}$, where $\mathrm{A}_{\mathrm{Li}}=\log \varepsilon(\mathrm{Li})$. However, the effect of age is related to the effect of rotation and chromospheric activity, since young stars rotate faster and exhibit more violent chromospheric activities than old stars.

The lithium behavior in dwarfs with and without planets has been well studied, but there are conflicting conclusions. Some studies (e.g. Israelian et al. 2004, Takeda \& Kawanamoto 2005, Chen et al. 2006, Israelian et al. 2009) have found that lithium abundance dilution is slightly higher in stars with planets compared to those without them. Also, other studies (e.g. Luck \& Heiter 2006, Baumann et al. 2010) found that there is no difference in lithium abundance between stars with and without planets. However, such kind of researches have not been carried out on a large sample of giants. A study of lithium abundances derived from a large homogeneous sample is desired, which allows a better understanding of lithium depletion. With more and more planets (23 planets up until now) released from the Okayama Planet Search program (Sato et al. 2003) and the Xinglong Planet Search program (Liu et al. 2008), this gives us a chance to systematically study the effect of planets on the lithium abundance for $\mathrm{G} / \mathrm{K}$ giants.

As is known, chemical composition is different in thin and thick disk stars in the solar neighborhood, particularly for the $\alpha$-elements and oxygen (e.g. Bensby 2005; Reddy et al. 2006). But whether the lithium abundance is different in thin/thick-disk stars was not known before Ramírez et al. (2012), who investigated the behavior of lithium abundance in thin-/thick-disk stars among dwarfs and subgiants. They observed a difference in lithium 
abundance between thin- and thick-disk stars, reflecting different degrees of lithium depletion rather than differences in lithium enrichment of the interstellar medium. With a catalog supplemented from the literature (Luck \&Heiter 2007, hereby Luck07), our sample has been enlarged to 565 giants, which can be used to explore the different properties of thin-/thickdisk giants.

In this paper, we analyze the lithium abundance for $378 \mathrm{G} / \mathrm{K}$ giants. The purpose of this work is to investigate the behavior of the lithium abundance as a function of metallicity, effective temperature, and stellar rotation, as well as to explore if there is any difference between giants with and without planets. The paper is organized as following: section 2 gives the sample selection and observational data; section 3 describes the method of analysis; section 4 presents our discussion; section 5 confirms the lithium abundance as a function of metallicity and effective temperature and the effect of thin-/thick-disk star in a sample of literature compilation 565 giants; finally the results are summarized in the last section.

\section{Sample selection and observational data}

The sample stars analyzed here are comprised of 321 giants from the Okayama Planet Search Program (Sato et al. 2003) and 57 giants from the Xinglong Planet Search Program (Liu et al. 2008). Both programs aim to detect planets around intermediate-mass G-type (and early K-type) giants. The spectra were taken with the HIgh Dispersion Echelle Spectrograph (HIDES) at Okayama Astrophysical Observatory (OAO), which was equipped at the coude focus of the $1.88 \mathrm{~m}$ telescope during 2008-2010. Before October 2008, one CCD system with wavelength coverage of 5000-6200 $\AA$ was used, and after that the new mosaic 3 CCD system with wavelength coverage of 4000-7540 $\AA$ replaced it. For all the 378 stars, several spectra were obtained with the iodine absorbtion cell in the planet search program. In such cases, two spectra with the highest S/N (all higher than 200 per pixel) were selected for each star. Meanwhile, spectra without the iodine cell were obtained for 71 stars as a comparison sample. The $\mathrm{S} / \mathrm{N}$ of these pure stellar spectra ranges from 100 to 250 . A slit width of $200 \mu \mathrm{m}$ was adopted, corresponding to a spectral resolution of 67,000 .

The 321 giants from the Okayama Planet Search Program have been analyzed by Takeda et al. (2008), who determined the stellar parameters, iron abundances and rotational velocities. The 57 giants from the Xinglong Planet Search Program were studied by Liu et al. (2010), in which they have derived the stellar parameters and iron abundances. Based on the latter work, it was shown that the stellar parameters of the sample of Liu et al. (2010) are located in the same region of the Hertzsprung-Russel(H-R) diagram as those of Takeda et al. (2008), thus we can consider the 378 stars as a uniform sample in the following analysis. 


\section{Analysis}

The ODF line-blanketed analysis with local thermodynamic equilibrium (LTE) is performed based on the model atmosphere MAFAGS developed and discussed by Fuhrmann et al. (1997). The stellar parameters of our sample are taken from Takeda et al. (2008) and Liu et al. (2010). Although the methods to determine stellar parameters are different, the results are quite consistent (see Liu et al. 2010). We adopt the oscillator strengths from the NIST database, with $\log g f=0.002$ and -0.299 for the 6707.76 and $6707.91 \AA$ lines, respectively. The collisional broadening parameters which describe the van der Waals interaction with the hydrogen atoms are taken from Barklem et al. (1998).

The lithium abundances are determined using the spectral synthesis method with the IDL/Fortran SIU software package developed by Reetz (1993). The synthetic spectrum is represented by a single Gaussian profile, with the combined broadening effects of stellar rotation, macroturbulence and the instrument profile. The lithium abundances are obtained until a best fit is reached between the synthetic and observed spectra. The contribution of FeI $6707.46 \AA$ line has been included. Considering that the $\mathrm{S} / \mathrm{N}$ of all the spectra with the iodine absorption cell are higher than 200, the noise in the spectra account for less than 0.5 percent of the continuum spectra. We therefore set the detection limit as a $3 \sigma$ error, i.e., 1.5 percent of the continuum. For those stars whose lithium line profile is not clear enough but stronger than the detection limit, a best fit value is given and the star is marked as an undistinguished detection. The best fits obtained between observed and synthetic spectra for HD116292 (with clear detection of lithium), HD62345 (with undistinguished detection of lithium), and HD4398 (with only upper limit detected) are shown in Figure 1 as typical cases for our sample.

\subsection{Lithium abundance comparison for stars with $I_{2}$ and without $I_{2}$}

For the purpose of precise radial velocity measurements, the spectra were taken with an iodine absorption cell placed within the optical beam path, thus the stellar spectral lines located in the region of 5000-6300 $\AA$ were heavily blended with the absorption features of $\mathrm{I}_{2}$. The effect on chemical abundance measurement caused by $\mathrm{I}_{2}$ in the spectral region

redder than $6300 \AA$ is very weak. It is shown that the uncertainty of equivalent width caused by $\mathrm{I}_{2}$ around $6700 \AA$ is estimated to be $2 \mathrm{~m} \AA$ (Wang et al. 2011). We have also obtained both the pure stellar spectra and $\mathrm{I}_{2}$ superposed spectra for 71 stars, and compared the lithium abundance derived from these two types of spectra to clarify the pollution of $\mathrm{I}_{2}$ in the abundance of lithium for this study. Table 1 presents the signal to noise ratio and lithium abundance for the 71 comparison targets determined from both the pure stellar 

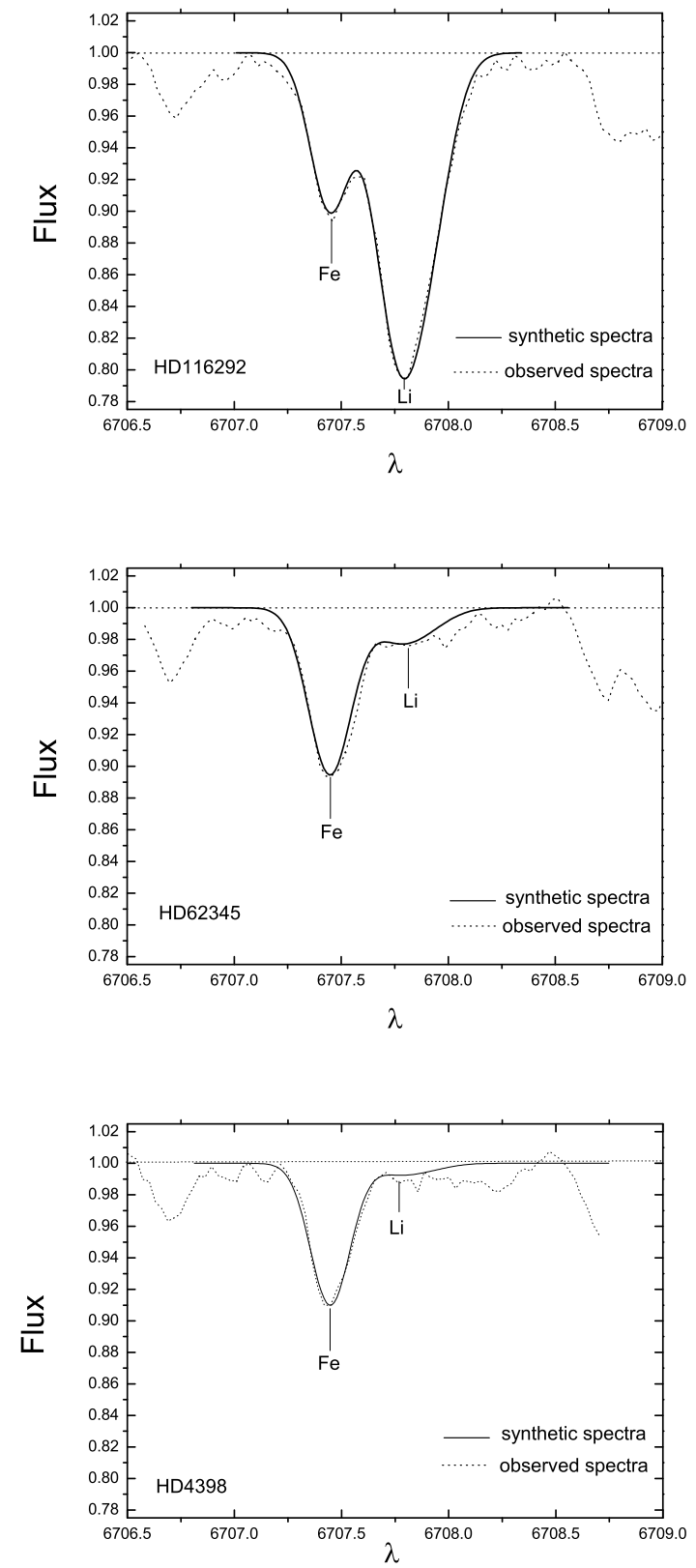

Fig. 1. - The best fits obtained between observed and synthetic spectra for HD116292, HD62345, and HD4398 (from top to bottom respectively). 
spectra and $\mathrm{I}_{2}$ superposed spectra. Figure 2 compares the lithium abundances determined from pure stellar spectra and spectra with $\mathrm{I}_{2}$ for the 71 stars. We divide our targets into four groups: a sub-sample with very clear detections of $\mathrm{Li}$; a sub-sample with undistinguished detections; a sub-sample with upper limits; and a sub-sample hosting planets. The four groups are represented by red open dots, green open squares, blue filled triangles, and black stars in Figures 2, and 5-11. For the 71 stars, the lithium abundances can be determined for 44 stars (with clear or undistinguished detections), and for the other 27 stars, only upper limits of Li could be derived. The lithium abundances derived from the spectra with $\mathrm{I}_{2}$ are consistent with those derived from the pure stellar spectra quite well for stars with detected lithium abundances, giving a difference of $0.00 \pm 0.06$ dex, but have a larger dispersion for stars with upper limit. Since the upper limit of the lithium abundance of the sub-sample strongly depends on the quality of the spectrum, namely $\mathrm{S} / \mathrm{N}$, the large difference may be due to the lower $\mathrm{S} / \mathrm{N}$ of the pure stellar spectra. Therefore, we consider that the lithium abundances derived from the spectra with $\mathrm{I}_{2}$ can represent the true lithium value. It is obvious that, for stars with clear or undistinguished detection of Li, the lithium abundances derived from spectra with $\mathrm{I}_{2}$ agree well with those derived from the pure stellar spectra, with a difference of $0.00 \pm 0.06$ dex. Meanwhile, the comparison shows a larger dispersion for stars with upper limits, considering the fact that the upper limits strongly depend on the quality of the spectra. 
Table 1. Comparison of the signal to noise ratio and derived lithium abundance based on pure stellar spectra and spectra with $\mathrm{I}_{2}$ superposed for 71 stars.

\begin{tabular}{lrrrr}
\hline \hline Star & S/N $\mathrm{N}_{\mathrm{Li} \text { (pure) }}$ & $\mathrm{S} / \mathrm{N}_{\mathrm{Li}}$ & $\mathrm{A}_{\mathrm{Li}(\text { pure })}$ & \multicolumn{1}{c}{$\mathrm{A}_{\mathrm{Li}}$} \\
& & & & \\
\hline HD 9408 & 385 & 305 & $<-0.06$ & $<-0.01$ \\
HD 11949 & 97 & 283 & 0.47 & 0.43 \\
HD 13994 & 222 & 282 & $<0.22$ & $<0.32$ \\
HD 15779 & 323 & 336 & 0.23 & 0.38 \\
HD 16400 & 279 & 276 & 0.22 & 0.22 \\
HD 18970 & 320 & 424 & 0.26 & 0.21 \\
HD 22675 & 341 & 263 & 0.62 & 0.57 \\
HD 27371 & 311 & 459 & 1.09 & 1.13 \\
HD 27697 & 340 & 359 & 1.05 & 1.07 \\
HD 28305 & 278 & 424 & 0.80 & 0.85 \\
HD 28307 & 321 & 320 & 1.20 & 1.23 \\
HD 36079 & 242 & 368 & $<0.23$ & $<0.23$ \\
HD 39007 & 367 & 249 & 0.36 & 0.46 \\
HD 41361 & 333 & 297 & 1.23 & 1.25 \\
HD 43039 & 325 & 296 & $<0.11$ & $<-0.04$ \\
HD 45410 & 289 & 291 & $<0.10$ & $<0.20$ \\
HD 50522 & 350 & 344 & 0.44 & 0.42 \\
HD 54810 & 274 & 281 & $<-0.12$ & $<-0.09$ \\
HD 57727 & 242 & 339 & 1.34 & 1.34 \\
HD 61363 & 273 & 320 & $<-0.03$ & $<-0.03$ \\
HD 62509 & 354 & 222 & 0.79 & 0.82 \\
HD 71115 & 242 & 305 & 0.78 & 0.83 \\
HD 71369 & 363 & 335 & 0.84 & 0.71 \\
HD 79181 & 218 & 261 & 0.24 & 0.29 \\
HD 81688 & 244 & 321 & $<-0.11$ & $<-0.11$ \\
HD 82210 & 312 & 225 & 1.18 & 1.17 \\
HD 84441 & 357 & 354 & $<0.44$ & $<0.44$ \\
HD 91190 & 215 & 351 & 0.55 & 0.62 \\
HD 92125 & 242 & 339 & $<0.61$ & $<0.71$ \\
HD 93291 & 194 & 241 & $<0.38$ & $<0.28$ \\
HD 101484 & 321 & 0.16 & 0.24 \\
& & & & 0.41
\end{tabular}


Table 1-Continued

\begin{tabular}{lrrrr}
\hline \hline Star & S/N $\mathrm{N}_{\mathrm{Li}(\text { pure })}$ & $\mathrm{S} / \mathrm{N}_{\mathrm{Li}}$ & $\mathrm{A}_{\mathrm{Li}(\text { pure })}$ & \multicolumn{1}{c}{$\mathrm{A}_{\mathrm{Li}}$} \\
& & & & \\
\hline HD 103484 & 172 & 247 & $<0.37$ & $<0.37$ \\
HD 104979 & 214 & 361 & $<0.13$ & $<0.23$ \\
HD 104985 & 190 & 259 & $<-0.17$ & $<-0.07$ \\
HD 106057 & 215 & 270 & 0.7 & 0.75 \\
HD 106714 & 233 & 309 & 0.75 & 0.72 \\
HD 107383 & 209 & 382 & 0.48 & 0.55 \\
HD 109317 & 191 & 263 & $<0.33$ & $<0.48$ \\
HD 113226 & 207 & 463 & 0.50 & 0.6 \\
HD 120048 & 193 & 224 & 1.39 & 1.44 \\
HD 120420 & 146 & 268 & 0.64 & 0.60 \\
HD 136512 & 319 & 319 & 0.34 & 0.34 \\
HD 136956 & 353 & 258 & 0.66 & 0.64 \\
HD 138905 & 306 & 479 & 1.27 & 1.33 \\
HD 142091 & 320 & 379 & $<0.33$ & $<0.33$ \\
HD 146791 & 387 & 370 & $<0.36$ & $<0.41$ \\
HD 148604 & 245 & 255 & 1.20 & 1.18 \\
HD 154084 & 314 & 344 & $<0.27$ & $<0.17$ \\
HD 167042 & 234 & 302 & $<0.23$ & $<0.28$ \\
HD 167768 & 299 & 232 & 0.18 & 0.13 \\
HD 188310 & 339 & 318 & $<0.20$ & $<0.10$ \\
HD 188650 & 310 & 324 & 1.01 & 0.91 \\
HD 199665 & 391 & 309 & 0.48 & 0.53 \\
HD 212496 & 305 & 351 & $<0.10$ & $<-0.07$ \\
HD 219139 & 284 & 287 & 0.19 & 0.19 \\
HD 221345 & 308 & 341 & $<0.19$ & $<0.14$ \\
HD 6482 & 263 & 264 & 0.55 & $<0.55$ \\
HD 6557 & 160 & 278 & $<0.11$ & $<0.11$ \\
HD 22799 & 243 & 285 & 0.19 & 0.09 \\
HD 23183 & 195 & 249 & 0.34 & 0.34 \\
HD 41125 & 280 & 251 & 0.59 & 0.54 \\
HD 82638 & 221 & 229 & $<0.20$ & $<0.20$ \\
HD 130025 & 316 & 244 & 0.59 & 0.44
\end{tabular}




\subsection{Error analysis}

The error in determining the lithium abundance with the method of spectrum synthesis mainly comes from two aspects: the systematic errors introduced by the atmospheric parameters and the uncertainty in the stellar continuum which could lead to differences in the lithium abundance.

It is known that the lithium abundance is very sensitive to the effective temperature, but the effects of surface gravity, iron abundance and microturbulence velocity are negligible. We calculate the variance of the lithium abundances due to changes of $100 \mathrm{~K}$ in effective temperature, 0.1 dex in surface gravity, 0.1 dex in metallicity, and $0.2 \mathrm{~km} \mathrm{~s}^{-1}$ in microturbulence velocity for a typical star HD12339. These values correspond to the maximum error of stellar parameters of our sample (see Takeda et al. 2008, Liu et al. 2010). The typical error of $100 \mathrm{~K}$ in effective temperature for giants will lead to a deviation of 0.1 dex in the lithium abundance, however the uncertainty of the lithium abundance is negligible by changes of 0.1 dex in surface gravity, 0.1 dex in metallicity, and $0.2 \mathrm{~km} \mathrm{~s}^{-1}$ in microturbulence velocity. Therefore, the error of $\mathrm{Li}$ abundance due to uncertainties in stellar parameters is at the level of 0.1 dex.

As for uncertainty in the stellar continuum, since all spectra used to derive lithium abundance are with $\mathrm{S} / \mathrm{N}$ higher than 200, the abundance error caused by location in the continuum is estimated to be less than 0.5 per cent in the worst case, which results in an error of lithium abundance of \pm 0.1 dex at most. The overall uncertainty in lithium abundance for our sample is less than 0.14 dex.

\subsection{Non-LTE correction}

According to Lind et al. (2009), the non-LTE effect can be very large on lithium abundance in giants. We perform the non-LTE calculations of lithium based on the grid and interpolation code by Lind et al. (2009). For stars whose upper limits of Li abundances are very small, the correction cannot be determined from the code because the corresponding equivalent widths are beyond the grids. It is shown in the grids that the non-LTE correction becomes independent of $\mathrm{A}_{\mathrm{Li}}$ when it is less than 0.6 dex; therefore, we take the minimum value from the grid and obtain the corresponding correction. For a few stars with microturbulence

velocity of $0.9 \mathrm{~km} \mathrm{~s}^{-1}$, which is out of the range of microturbulence velocity in the grid, the correction corresponding to $1.0 \mathrm{~km} \mathrm{~s}^{-1}$ is then adopted. Considering that the calculation for non-LTE correction is related to effective temperature, metallicity, surface gravity and microturbulence, the non-LTE corrections are thus plotted against these four parameters 
Table 1-Continued

\begin{tabular}{crrrr}
\hline \hline Star & S/N $\mathrm{N}_{\mathrm{Li}(\text { pure })}$ & $\mathrm{S} / \mathrm{N}_{\mathrm{Li}}$ & $\mathrm{A}_{\mathrm{Li}(\text { pure })}$ & \multicolumn{1}{c}{$\mathrm{A}_{\mathrm{Li}}$} \\
& & & & \\
\hline HD 136366 & 212 & 199 & 0.57 & 0.62 \\
HD 141853 & 219 & 202 & $<0.04$ & $<0.14$ \\
HD 173398 & 323 & 279 & 0.51 & 0.51 \\
HD 173416 & 261 & 326 & 0.22 & 0.12 \\
HD 175679 & 263 & 265 & 0.99 & 0.97 \\
HD 206005 & 257 & 256 & $<0.18$ & $<0.23$ \\
HD 220465 & 223 & 265 & $<0.22$ & $<0.07$ \\
\hline \hline
\end{tabular}

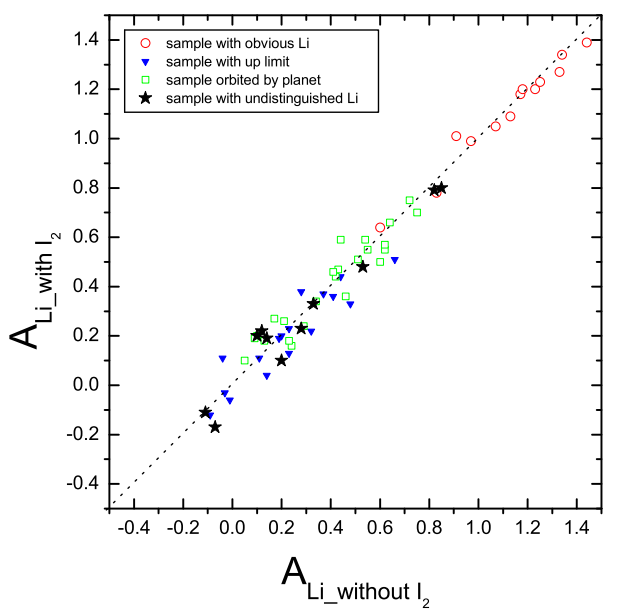

Fig. 2.- Comparison of the lithium abundances determined from the spectra with and without $\mathrm{I}_{2}$ for 71 comparison stars. The red open dots, filled squares, open triangles and stars represent the sub-sample groups with clear Li detection, with undistinguished Li detection, with Li upper limits and with planets, respectively. 
in Figure 3. As expected, the non-LTE correction is a function of effective temperature; the lower the temperature, the higher the correction value. However, the correction shows no clear correlation with metallicity, surface gravity or microturbulence velocity. Table 2 presents the star name, effective temperature, surface gravity, metallicity, stellar mass, lithium abundance or upper limit of the four sub-sample groups as described in the text, as well as the non-LTE correction. The range of non-LTE correction is 0.05-0.28 dex, with an average of 0.18 dex for most sample stars. Therefore, in $\mathrm{G}$ and $\mathrm{K}$ giants, the non-LTE effect on lithium abundance can not be ignored.

\subsection{Comparison with literatures}

We compare the derived lithium abundance with previous studies by Luck07, Lèbre et al. (2006) and Luck \& Wepfer (1995). Since the lithium abundance from these studies were derived based on LTE model, we also adopt our LTE results for comparison.

\section{Comparison with Luck07}

Based on a large sample of giants with high quality spectra, Luck07 has carried out precise parameter determination and abundance analysis on many elements. Three sets of parameters and corresponding lithium abundances are determined for 298 nearby giants, of which 96 stars are in common with our work. The three sets of parameters include physical parameters, and two sets of spectroscopic parameters which based on different stellar atmospheric models (MARCS and MARCS75). Since the lithium abundance is more sensitive to effective temperature than to other parameters, we compare the three sets of effective temperature with this study (hereby referred to Liu2013) to get the best fit of effective temperature and the corresponding lithium abundances. From Figure 4(a), the effective temperatures from physical data (filled dots) are consist with ours with the average difference of $6 \pm 79 \mathrm{~K}$ for 96 common stars, and hence, we compare the lithium abundances based on physical parameters (filled dots) with our results in Figure 4(b). For the 43 stars with $\mathrm{Li}$ detections in both studies, our lithium abundances are quite close to those of Luck07

with the average difference being $0.09 \pm 0.13$ dex. For stars with upper limits of $A_{L i}<0$, the difference is larger. Taking into consideration of the following two comparisons with other studies, it is suggested that the negative lithium abundances seem to be underestimated by Luck07.

\section{Comparison with Lèbre et al. (2006)}

Another study on the lithium abundances of a large sample of giant stars is Lèbre et al. (2006), who derived lithium abundances for 145 bright giants using the spectrum synthesis 

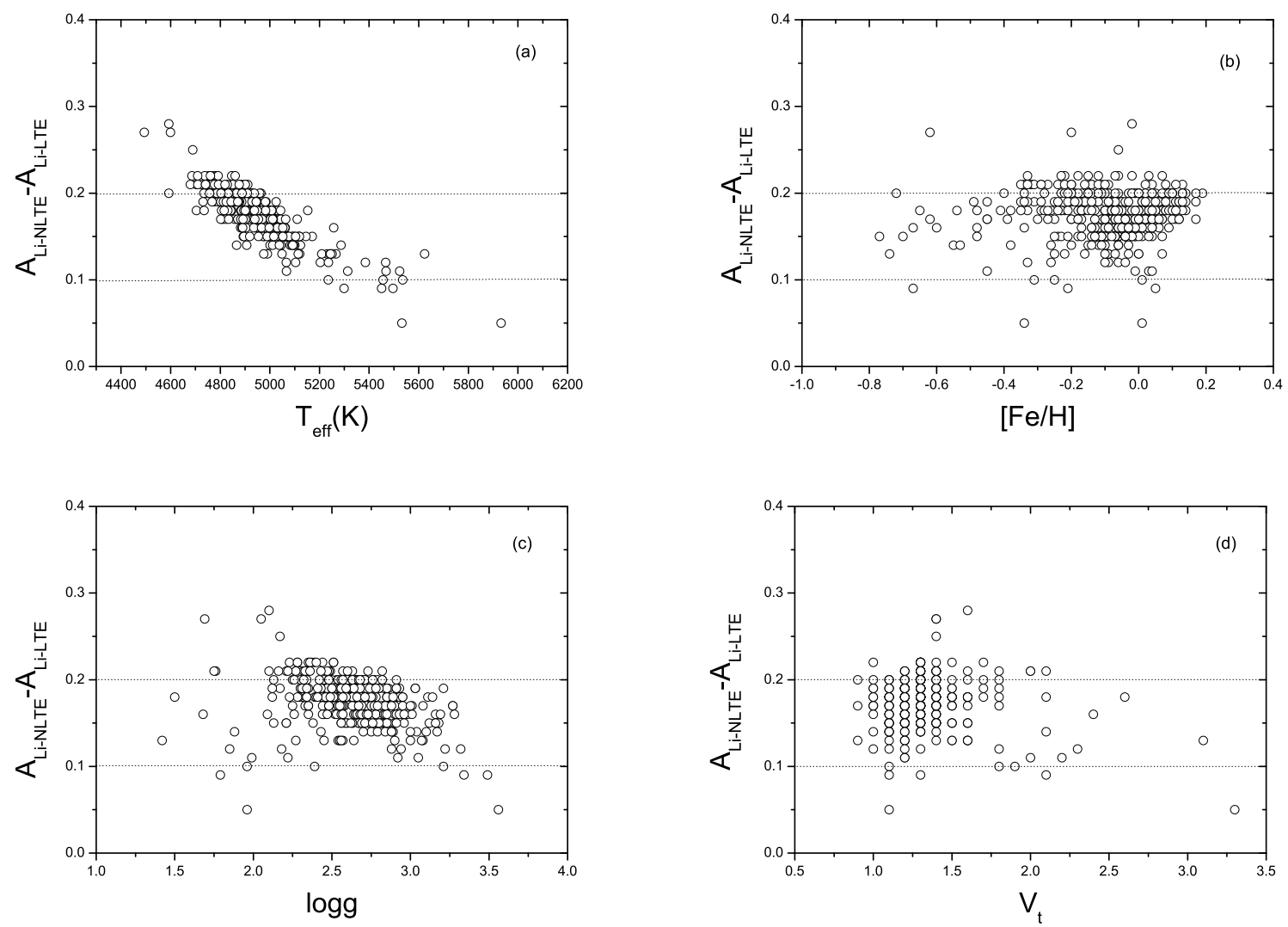

Fig. 3.- The non-LTE correction for lithium abundance against effective temperature (a), metallicity (b), surface gravity (c), and microturbulence velocity (d) for our sample. 

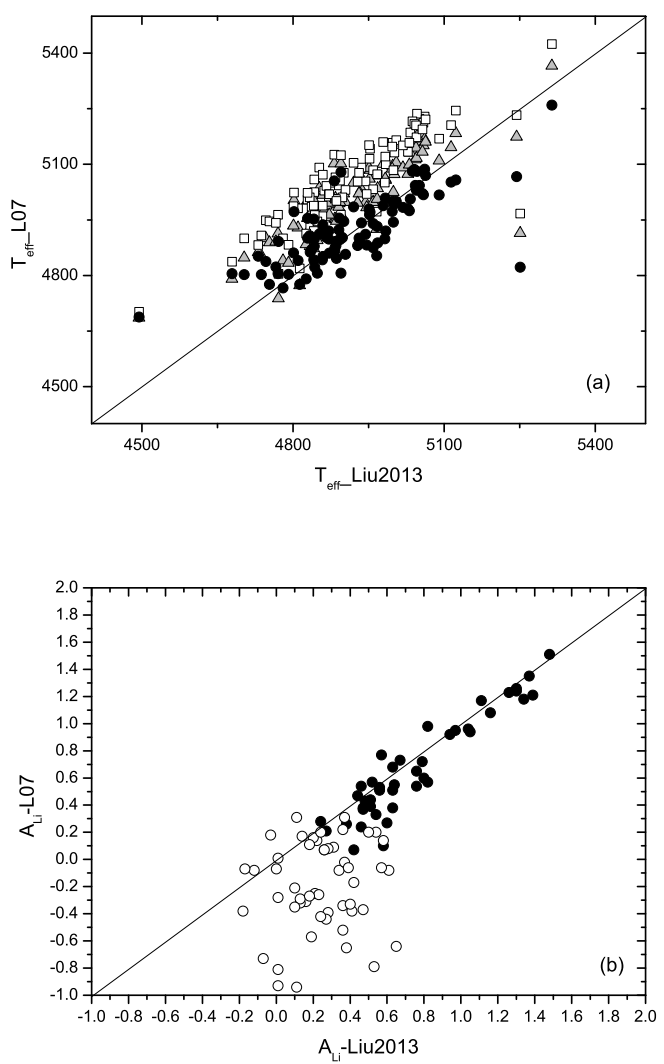

Fig. 4.- Comparisons of effective temperature and lithium abundances between Luck \& Heiter (2007) and this work. Filled dots, triangles and squares represent values from physical parameters, and spectroscopic parameters based on two sets of stellar atmospheric models. 
method. Comparisons of effective temperature and lithium abundances for 17 common stars with our work are presented in Table 3, including the effective temperature, lithium abundance, metallicity and the difference of the two works as well. It is noticed that the lithium abundances are in good agreement, within the measured errors for all common stars. The differences in effective temperature and lithium abundance are $53.5 \pm 121 \mathrm{~K}$ and $0.29 \pm$ 0.16 dex respectively; however, the latter is limited to only five stars showing Li detection in both works. The difference in lithium abundance for three stars, HD27022, HD65228 and HD76494, is relatively large, with values of $0.36,0.37$, and 0.46 respectively. The difference for HD27022 should come from difference in iron abundance, and that for HD65228 mainly

comes from difference in effective temperature. The large difference for HD76494 remains unclear; however, the lithium abundance for these three stars agree with those from Luck \& Wepfer (1995), with the average difference being less than 0.1 dex. Large differences are also seen for HD71115 and HD159181, which we have referred to undistinguished detection, while Lèbre et al. (2006) has assigned a uniform upper limit of 0.4 dex for both of them. Since the spectra from Lèbre et al. (2006) have much lower S/N (higher than 50) and lower resolution (45000) than ours, the large difference probably comes from relatively low quality of spectra in Lèbre et al. (2006). The lithium abundance of HD71115 from Lèbre et al. (2006) is remarkably different from that from Liu2013, but the value from Luck \& Wepfer (1995) agrees very well with our result.

\section{Comparison with Luck \& Wepfer (1995)}

Luck \& Wepfer (1995) have determined the stellar parameters and lithium abundances for 38 $\mathrm{F} / \mathrm{G}$ bright field giants, in which there are 15 stars in common with our sample. Among these 15 stars, 9 have clear Li detections in both works. The differences in effective temperature and lithium abundance are $11.5 \pm 71 \mathrm{~K}$ (for 15 stars) and $0.04 \pm 0.10$ dex (for 9 stars) respectively, which indicates a good agreement between these two studies. Comparisons of the above measurements are presented in Table 4. Note that the differences of HD36079 and HD92125 are quite large; they are assigned upper limits in our work, but with detected abundances from Luck \& Wepfer (1995). The reason still remains unclear, although we have carefully checked the spectra and parameters.

\section{Results and discussion}

The lithium abundance is supposed to depend on metallicity, effective temperature, stellar mass, age, stellar rotation and chromospheric activity for giants. However, the effect varies from different samples and different studies. In this work, we study the behavior of lithium abundance as a function of $[\mathrm{Fe} / \mathrm{H}], \mathrm{T}_{\text {eff }}$, and stellar rotation, as well as whether 
stars that host planets can affect their lithium abundances. We know that there are large uncertainties in stellar mass and age for red giants and red clump giants from the evolutionary tracks, hence we do not investigate the relations of lithium abundance against stellar mass and age in this study.

\subsection{Lithium abundance versus $[\mathrm{Fe} / \mathrm{H}]$}

The lithium abundance versus metallicity for our sample is plotted in Figure 5. The open dots, open squares, open triangles and stars respectively represent the sub-sample with clear Li detections, with undistinguished Li detections, with upper limits of lithium abundance, and with planets. The metallicity of our sample ranges from -0.8 to 0.2 , but in the lowermetallicity region with $[\mathrm{Fe} / \mathrm{H}]<-0.4$, only a few stars show $\mathrm{Li}$ detections. However, for those with $[\mathrm{Fe} / \mathrm{H}]>-0.4$, about half of them have Li detections.

According to theoretical models, the surface lithium is diluted by a factor of 28 to 61 for Red Giant Branch (RGB) stars with a mass of 1-5 $\mathrm{M}_{\odot}$ (Iben 1965, 1966, 1967a,b). These depletions correspond to a decrease in lithium abundance of 1.8-1.4 dex relative to the assumed initial value from the main sequence. However, some works based on observation found that the depletion is more severe than the theoretical prediction. For instance, Brown et al. (1989) derived an average lithium abundance lower than 0.0 dex for 891 late-G to $\mathrm{K}$ giants, which is at least 1.5 dex below the predicted value; Luck \& Wepfer (1995) found that lithium is depleted by a factor of 100 to 1000 for bright giants; and de Laverny et al. (2003) found a dilution by a factor of at least 600 for G0III type giants with a mass of 2 to $3 \mathrm{M}_{\odot}$. In our sample, Li depletion reached a factor of 770 for objects with Li detections compared with the current interstellar medium value of $\mathrm{A}_{\mathrm{Li}} \approx 3.3$, which is far more diluted than the theoretical prediction. The over depletion was also found by other studies focusing on giants (eg. Brown et al. 1989, De Medeiros et al. 2000, de Laverny et al. 2003). If the over depletion is due to an extra-mixing mechanism existed in red giants/red clump giants, the $[\mathrm{C} / \mathrm{Fe}]$ ratio, which indicates the degree of evolution-induced envelope mixing, would also reflect this difference; therefore, a correlation may be expected between $\mathrm{A}_{\mathrm{Li}}$ and $[\mathrm{C} / \mathrm{Fe}]$, which is not shown in Figure 6. From literatures we know that early-F type $\operatorname{stars}\left(\sim 1.5 \mathrm{M}_{\odot}\right)$ show a considerable diversity in $\mathrm{A}_{\mathrm{Li}}$ (e.g. Boesgaard \& Tripicco 1986). For A type stars $(\sim 2$ $\left.\mathrm{M}_{\odot}\right), \mathrm{A}_{\mathrm{Li}}$ is ranging from normal to undetected value (e.g. Takeda et al. 2012). For rapidly rotating A-B type stars $\left(2-4 \mathrm{M}_{\odot}\right)$, it is very hard to detect the surface Li value. This is probably because in early type stars, surface Li is depleted because of the envelope mixing due to meridional circulation. It is more convincing that Li deficiency may have already

existed in the main sequence phase for many $1.5-5 \mathrm{M}_{\odot}$ stars and was carried over to the 
evolved $\mathrm{G} / \mathrm{K}$ giants.

Except for three Li-rich stars with $\mathrm{A}_{\mathrm{Li}}>1.7$, the lithium abundance is increasing towards a higher metallicity in Figure 5. The dispersion of $\mathrm{Li}$ abundance at higher metallicities is very large (with a maximum of about $2.0 \mathrm{dex}$ ).

\subsection{Li-rich stars and Li-normal stars}

As predicted by theoretical models, typical RGB stars, with solar metallicity and stellar mass between 1.0 to $5.0 \mathrm{M}_{\odot}$ and which have undergone the first dredge up stage, are expected to show $\mathrm{A}_{\mathrm{Li}}<1.8-1.4$ dex. We have chosen the value of $\mathrm{A}_{\mathrm{Li}}>1.7 \mathrm{dex}$, and 1.3-1.7 dex as the standard to define a star as being abnormally Li-rich or Li-normal, as suggested by some investigations (e.g. Brown et al. 1989, Gonzalez et al. 2009). Among all samples, the three stars HD65228, HD212430 and HD102845 show $\mathrm{A}_{\mathrm{Li}}>1.7$, and are thus classified as Li-rich giants. There are 36 stars that exhibit the expected lithium abundances $\left(1.3 \leq \mathrm{A}_{\mathrm{Li}} \leq 1.7\right)$, i.e. Li-normal stars, and the remaining Li-depletion stars show an abundance of $\mathrm{A}_{\mathrm{Li}}<1.3$. Some researches (e.g. Brown et al. 1989, Charbonnel \& Balachandran 2000, Ramírez et al. 2012) show that the previous results indicate the fraction of Li-rich giants is less than $1 \%$, which agrees well with our results. To check the mass and evolutionary status of the Li-rich and Li-normal giants, we plot their positions in H-R diagram in Figure 7, by adopting the stellar evolutionary tracks of YY (Yi et al. 2003) with the mean metallicity $\mathrm{Z}=0.008$ $([\mathrm{Fe} / \mathrm{H}]=-0.4$, blue line $)$ and solar metallicity $\mathrm{Z}=0.02([\mathrm{Fe} / \mathrm{H}]=0$, green line $)$. The chosen mass range is 1.0 to $5 \mathrm{M}_{\odot}$, but most of them lie within $2-4 \mathrm{M}_{\odot}$. Three sizes of the symbols correspond to Li-rich, Li-normal and Li-depletion giants. Li-rich giants with $\mathrm{A}_{\mathrm{Li}}>1.7$ are represented by the largest, filled symbol, and 36 Li-normal giants with $1.3 \leq \mathrm{A}_{\mathrm{Li}} \leq 1.7$ by mid-size symbol. As a result of selection strategy in our sample, i.e., only late $\mathrm{G}$ and $\mathrm{K}$ giants are selected for planet search program, the majority of stars which are classified as RGBs are located in the same region of the H-R diagram. According to the statement of Charbonnel \& Balachandran(2000), the Li production phase is very short, which supports the result of observations that less than $1 \%$ giants are Li-rich, as confirmed both in previous studies and our work.

HD65228, possessing the highest lithium abundance in our sample, is located on the blue side of the Hertzsprung gap. This star just began to develop a convective envelope, and a high lithium abundance is expected by the standard theory prediction. For HD212430 and HD102845, they are located in the clump region of the H-R diagram, hence there are two possible scenarios to explain such high Li abundances (Brown et al. 1989). One is that these two stars might be the first ascent giants, which are partly evolved, and assumed to 
Table 2. The stellar parameters and lithium abundances of 378 red giants

\begin{tabular}{lrrrrrrrrrr}
\hline \hline \multicolumn{1}{c}{ Star } & $\mathrm{T}_{\text {eff }}(\mathrm{K})$ & $\log g$ & {$[\mathrm{Fe} / \mathrm{H}]$} & $M^{*}\left(\mathrm{M}_{\odot}\right)$ & A1 & A2 & A3 & A4 & $\Delta$ NLTE \\
& & & & & & & & & \\
\hline HD87 & 5072 & 2.63 & -0.07 & 2.74 & 0.99 & - & - & - & 0.15 \\
HD360 & 4850 & 2.62 & -0.08 & 2.34 & - & 0.4 & - & - & 0.19 \\
HD448 & 4780 & 2.51 & 0.03 & 2.25 & - & - & 0.36 & - & 0.22 \\
HD587 & 4893 & 3.08 & -0.09 & 1.58 & - & 0.37 & - & - & 0.17 \\
HD645 & 4880 & 3.03 & 0.07 & 1.95 & 0.63 & - & - & - & 0.19 \\
$\ldots \ldots \ldots \ldots .$. & & & & & & & & & & \\
$\ldots$
\end{tabular}

Note. - Table 2 is published in its entirety in the electronic edition of The Astrophysical Journal. A portion is shown here for guidance regarding its form and content.

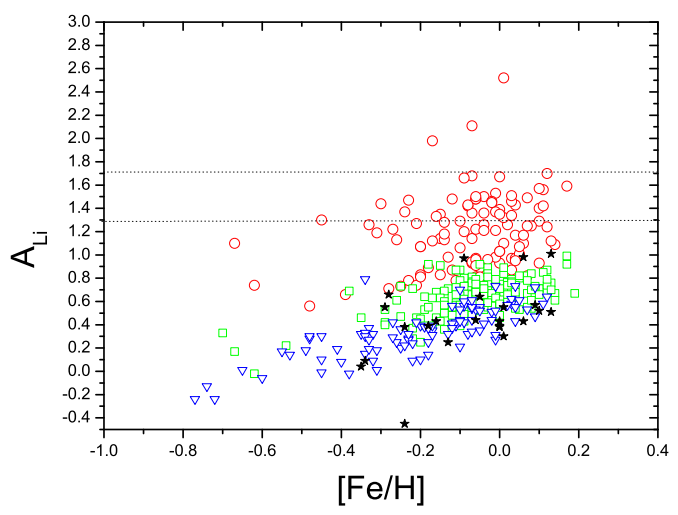

Fig. 5.- Lithium abundance versus metallicity. The symbols are the same as in Figure2. Two dash lines divide the regions corresponding to Li-rich, Li-normal and Li-depletion. 
Table 3. The comparison of effective temperature and lithium abundances with Lèbre et al. (2006) for 17 common stars.

\begin{tabular}{cccrrrrrrr}
\hline \hline Star & \multirow{2}{*}{$\mathrm{T}_{\text {eff }} \mathrm{T}_{\text {eff 06 }}$} & \multicolumn{1}{c}{$\mathrm{A}_{\mathrm{Li}}$} & $\mathrm{A}_{\mathrm{LiL06}}$ & {$[\mathrm{Fe} / \mathrm{H}]$} & {$[\mathrm{Fe} / \mathrm{H}]_{\mathrm{L} 06}$} & $\Delta \mathrm{T}_{\text {eff }}$ & $\Delta \mathrm{A}_{\mathrm{Li}}$ \\
& & & & & & & & \\
\hline HD 1367 & 4982 & 5150 & $<0.57$ & $<0.4$ & -0.01 & 0.00 & -168 & 0.17 \\
HD 27022 & 5314 & 5280 & 1.26 & 0.9 & -0.01 & -0.20 & 34 & 0.36 \\
HD 40801 & 4834 & 5000 & 0.46 & $<0.4$ & -0.17 & -0.30 & -166 & 0.06 \\
HD 65228 & 5932 & 5600 & 2.47 & 2.1 & 0.01 & 0.0 & 332 & 0.37 \\
HD 67447 & 4974 & 5000 & 1.3 & 1.2 & -0.06 & 0.00 & -26 & 0.1 \\
HD 71115 & 5062 & 5150 & 0.78 & $<0.4$ & -0.07 & -0.10 & -88 & $>0.38$ \\
HD 76219 & 4904 & 5000 & 0.43 & $<0.4$ & -0.15 & -0.20 & -96 & 0.03 \\
HD 76494 & 4828 & 4950 & 0.86 & 0.4 & -0.20 & -0.30 & -122 & 0.46 \\
HD 98839 & 4936 & 5101 & - & $<0.4$ & -0.05 & 0.00 & -74 & - \\
HD 106057 & 4956 & 5100 & $<0.76$ & $<0.4$ & -0.10 & -0.20 & -144 & 0.36 \\
HD 109379 & 5145 & 5150 & 1.06 & 0.9 & -0.01 & -0.3 & -5 & 0.16 \\
HD 119035 & 4816 & 4900 & $<0.28$ & $<0.4$ & -0.35 & -0.2 & -84 & -0.12 \\
HD 150030 & 4850 & 4900 & $<0.39$ & $<0.4$ & -0.09 & -0.20 & -50 & -0.01 \\
HD 159181 & 5153 & 5300 & 0.73 & $<0.4$ & -0.15 & 0.30 & -147 & $>0.33$ \\
HD 174980 & 5008 & 5150 & $<0.53$ & $<0.4$ & 0.10 & 0.10 & -142 & 0.13 \\
HD 206731 & 4936 & 4970 & $<0.39$ & $<0.4$ & -0.14 & -0.3 & -34 & -0.01 \\
HD 210807 & 5071 & 5000 & 0.63 & $<0.4$ & -0.10 & -0.10 & 71 & $>0.23$ \\
\hline \hline
\end{tabular}


Table 4. The comparison of effective temperature and lithium abundances with Luck \& Wepfer (1995) for 15 common stars.

\begin{tabular}{crrrrrr}
\hline \hline Star & $\mathrm{T}_{\text {eff }}$ & $\mathrm{T}_{\text {eff(L95) }}$ & \multicolumn{1}{c}{$\mathrm{A}_{\mathrm{Li}}$} & $\mathrm{A}_{\mathrm{Li}(\mathrm{L} 95)} \Delta \mathrm{T}_{\text {eff }}$ & $\Delta \mathrm{A}_{\mathrm{Li}}$ \\
& & & & & & \\
\hline HD 27022 & 5314 & 5275 & 1.26 & 1.25 & 39 & 0.01 \\
HD 36079 & 5209 & 5225 & $<0.23$ & 0.68 & -16 & $>-0.45$ \\
HD 65228 & 5932 & 5900 & 2.47 & 2.52 & 32 & -0.05 \\
HD 67447 & 4974 & 4825 & 1.3 & 1.15 & -51 & 0.15 \\
HD 71115 & 5062 & 5050 & 0.78 & 0.80 & 12 & -0.02 \\
HD 76219 & 4904 & 4875 & 0.43 & 0.31 & 29 & 0.12 \\
HD 76294 & 4844 & 4850 & 0.57 & 0.40 & -6 & 0.17 \\
HD 76494 & 4828 & 4900 & 0.86 & 1.00 & -72 & -0.14 \\
HD 84441 & 5385 & 5300 & $<0.44$ & 0.67 & 85 & $>-0.27$ \\
HD 92125 & 5468 & 5600 & $<0.61$ & 1.01 & -132 & $>-0.4$ \\
HD 99648 & 5002 & 4850 & 0.42 & 0.32 & 152 & 0.1 \\
HD 109379 & 5145 & 5125 & 1.06 & 1.03 & 20 & 0.03 \\
HD 144608 & 5266 & 5200 & $<0.43$ & 0.31 & 66 & $<0.12$ \\
HD 150030 & 4850 & 4775 & $<0.39$ & 0.26 & 75 & $<0.13$ \\
HD 214567 & 4989 & 5050 & $<0.27$ & -0.26 & -61 & $<0.53$ \\
\hline
\end{tabular}

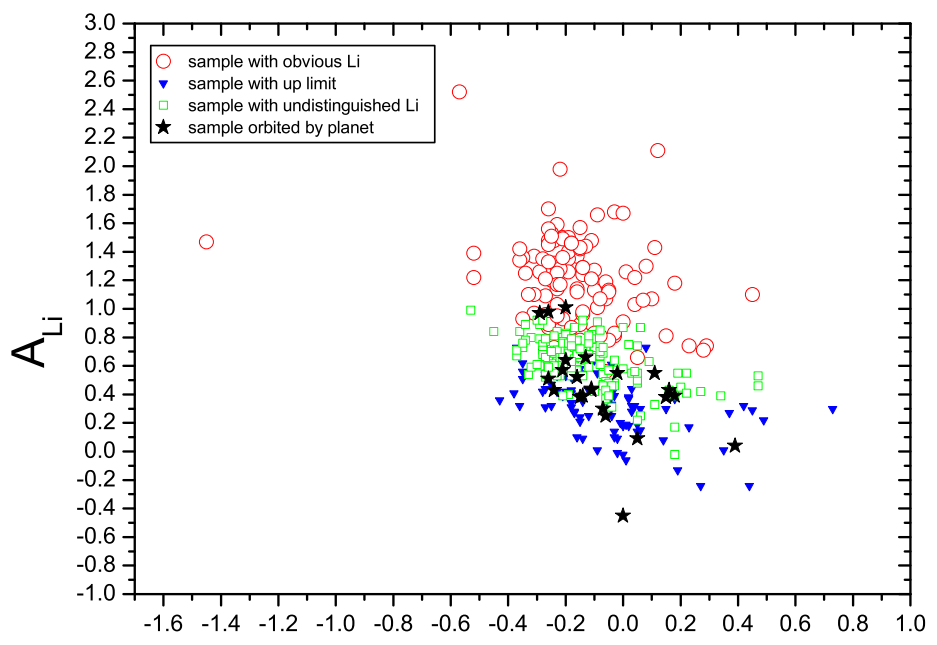

[C/Fe]

Fig. 6.- Lithium abundance versus [C/Fe]. The symbols are the same as in Figure2. 
have left the main sequence with their initial surface $\mathrm{Li}\left(\mathrm{A}_{\mathrm{Li}} \approx 3.3\right)$. The other explanation is that they have a special circumstance that show high surface Li abundances, which is called the Cameron-Fowler mechanism (Cameron \& Fowler 1971), and predicts that carbon isotopic ratios will decrease. Li production precedes the extra-mixing phase which connects the convective envelope with the $\mathrm{CN}$-burning region to produce the low ${ }^{12} \mathrm{C} /{ }^{13} \mathrm{C}$ ratio. The mixing result in the production of ${ }^{7} \mathrm{Li}$, which is then convected to the surface. We know that 96\% of the evolved stars show a low ${ }^{12} \mathrm{C} /{ }^{13} \mathrm{C}$ ratio (Charbonnel \& Do Nascimento 1998), which is in disagreement with standard predictions. Thus the most reasonable explanation is that they have undergone a period of Li-production. The 36 giants that have normal amounts of $\mathrm{Li}$, which contain about $10 \%$ of our sample, are supposed to show the present interstellar medium abundance of $\mathrm{A}_{\mathrm{Li}} \approx 3.3$, and have experienced the expected dilution phase during the first dredge-up phase. The surface lithium abundance decreases with respect to its value at the end of the main sequence by a factor of 30 to 60 , depending on the stellar mass and metallicity.

\subsection{Lithium abundance versus effective temperature}

Figure 8 shows that the lithium abundance is a function of temperature with the detection limit increasing when the effective temperature increases. Generally, from our sample, the lithium abundance increases towards a higher metallicity for our sample, but in the narrow range of 4800-5100 K, there is no clear correlation between lithium abundance and effective temperature. Previous studies (Wallerstein et al. 1994, De Medeiros et al. 2000, de Laverny et al. 2003) have demonstrated that the lithium abundance is a function of effective temperature. However, we also notice that, within the color index range of $0.8<\mathrm{B}-\mathrm{V}$ $<1.0$, the well established gradual decline of lithium abundances as a function of effective temperature is not clear in many studies (Wallerstein et al. 1994, De Medeiros et al. 2000). The lithium abundances span two orders at a given effective temperature for our sample, suggesting that the stellar evolution stage and mass vary with dilution (De Medeiros et al. 2000).

To get rid of the effect of population, we divide our sample into 3 groups by metallicity: $-0.8<[\mathrm{Fe} / \mathrm{H}] \leq-0.4,-0.4<[\mathrm{Fe} / \mathrm{H}] \leq-0.1$, and $-0.1<[\mathrm{Fe} / \mathrm{H}] \leq 0.2$; we then plot the lithium abundance versus effective temperature in Figure 9. To get the general behavior of lithium in red giants, the three Li-rich stars $\left(\mathrm{A}_{\mathrm{Li}}>1.7\right)$ HD65228, HD212430 and HD102845 are not plotted in these figures. It is clear that there is no correlation between the lithium abundance and effective temperature in the metallicity range of $-0.1<[\mathrm{Fe} / \mathrm{H}] \leq 0.2$, but with weak correlation in the metallicity range of $-0.4<[\mathrm{Fe} / \mathrm{H}] \leq-0.1$. Although there are 


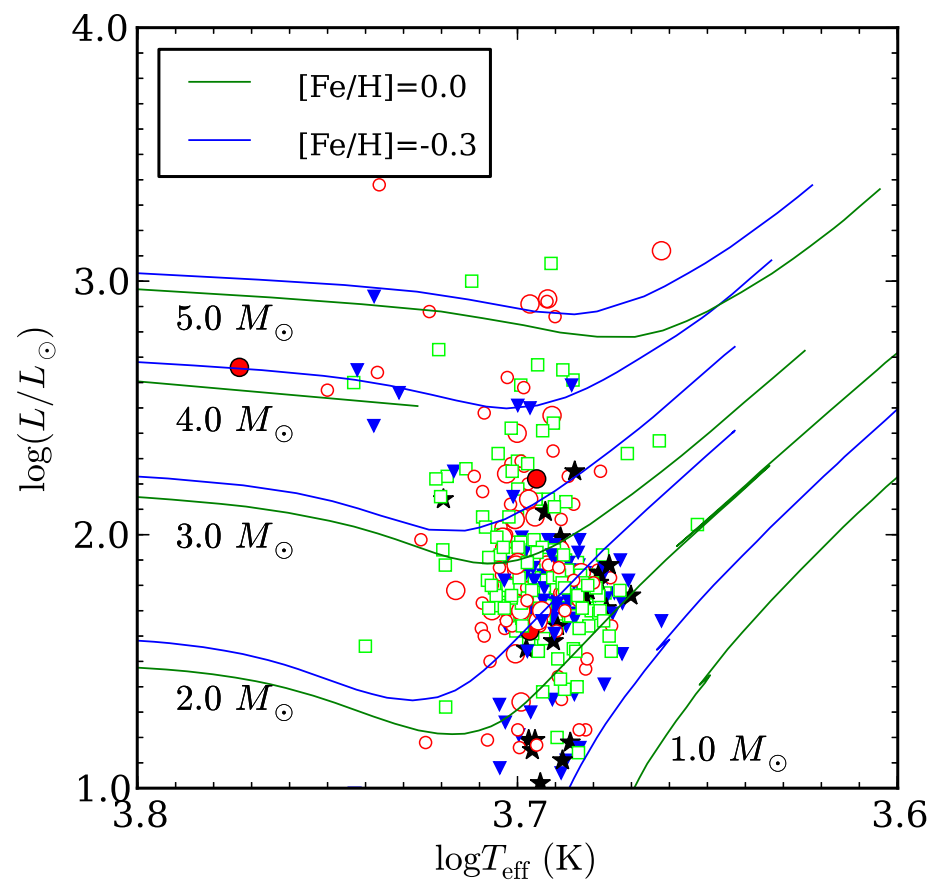

Fig. 7.- Lithium abundance in H-R diagram. The symbols are the same as in Figure2. Three sizes of the symbols correspond to Li-rich, Li-normal and Li-depletion giants.

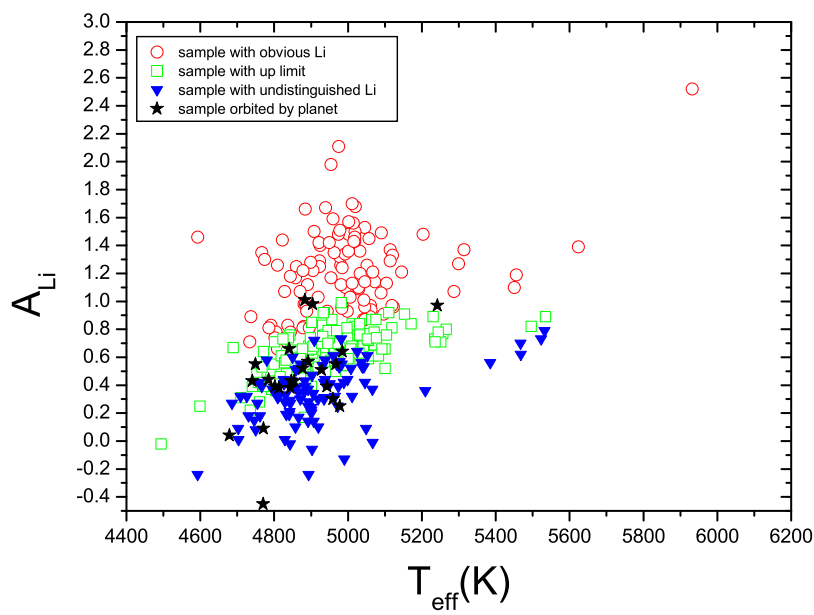

Fig. 8.- Lithium abundance versus effective temperature. The symbols are the same as in Figure2. 
only four stars with clear Li detections in the metallicity range of $-0.8<[\mathrm{Fe} / \mathrm{H}] \leq-0.4$, the lithium abundance as a function of effective temperature is quite clear.

\subsection{Lithium abundance versus rotational velocity}

The rotational velocities in this analysis for 321 giants are taken from Takeda et al. (2008), but the rotational velocity are not available for the other 57 giants in Liu et al. (2010). For these 57 giants, the total macrobroadening function is a convolution of instrumental broadening, rotation and macroturbulence, and can be derived from the process of deriving the lithium abundance, with a maximum value of $8 \mathrm{~km} \mathrm{~s}^{-1}$. Therefore, the rotational velocity should be no faster than $8 \mathrm{~km} \mathrm{~s}^{-1}$. Considering that there is no systematic difference between a sample of 321 and 378, we only compare the lithium abundance versus rotational velocity for the 321 sample giants in Figure 10. Since our sample is taken from the planet search program, which select stars with slow rotational velocity to ensure the spectral line will not be broadened too much, the rotational velocity for most of our sample is slower than $6 \mathrm{~km}$ $\mathrm{s}^{-1}$, and there are only a few between $6-11 \mathrm{~km} \mathrm{~s}^{-1}$. Therefore, it is not surprising that the lithium abundance shows no correlation with rotational velocity when it is smaller than 10 $\mathrm{km} \mathrm{s}^{-1}$. The spread in lithium abundance is large in the values of vsini $<10 \mathrm{~km} \mathrm{~s}^{-1}$, and only two stars show $10 \mathrm{~km} \mathrm{~s}^{-1}<$ vsini $<11 \mathrm{~km} \mathrm{~s}^{-1}$ in this sample. One of the latter presents the highest lithium abundance and the other has only been assigned with an upper limit. HD65228, the most Li-rich object, shows the highest rotational velocity of $10.28 \mathrm{~km} \mathrm{~s}^{-1}$, and it also shows the highest temperature in our sample. Previous studies (De Medeiros et al. 2000, de Laverny et al. 2003, Lèbre et al. 2006) found that the stars with higher or moderate rotational velocity (vsini $>10 \mathrm{~km} \mathrm{~s}^{-1}$ ) tend to show higher lithium abundances. Our results are consistent with the results from de Laverny et al. (2003), in which lithium abundance shows a wide spread at vsini $<4 \mathrm{~km} \mathrm{~s}^{-1}$.

Considering that many stars in our sample have an effective temperature ranging from 4800-5100 K, we plot the lithium abundance for stars with the temperature of 4800-5100 $\mathrm{K}$ against metallicity and rotational velocity in Figure 11, for the purpose of taking off the effect of effective temperature. We omit the three lithium rich giants $\left(A_{\mathrm{Li}}>1.7\right)$ in Figure 11 to show the general behavior of lithium in red giants. It becomes more clear that the lithium abundance is not correlated with metallicity, or rotational velocity in the temperature range of $4800-5100 \mathrm{~K}$. 

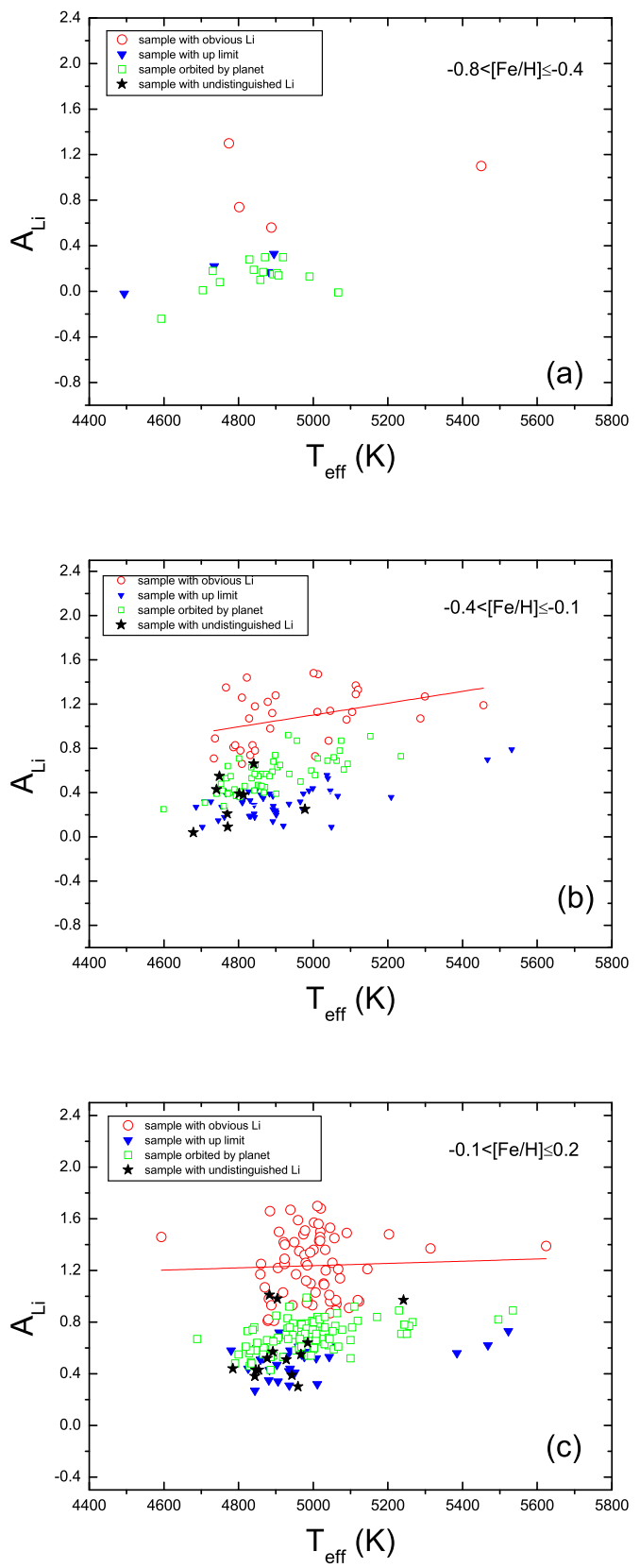

Fig. 9.- Lithium abundance against effective temperature in three different metallicity coverage. (a). $-0.8<[\mathrm{Fe} / \mathrm{H}] \leq-0.4$, (b) $-0.4<[\mathrm{Fe} / \mathrm{H}] \leq-0.1$, (c) $-0.1<[\mathrm{Fe} / \mathrm{H}] \leq 0.2$. The symbols are the same as in Figure2. 


\subsection{Lithium in planet-hosting giants and giants with not known giant planets}

Lithium abundances are believed to be important for potentially providing a better understanding of processes involved in the formation and evolution of planetary systems.

There are extensive studies on lithium in dwarfs that host planets and those without giant planets; however, there are conflicting results. It has been found in many studies (Israelian et al. 2004, Takeda \& Kawanomoto 2005, Chen et al. 2006, Israelian et al. 2009) that dwarfs that host planets, with effective temperature ranging from 5600 to $5850 \mathrm{~K}$, exhibit severely depleted Li. A large fraction of the comparison sample only shows partially inhibited depletion. However, some studies (e.g. Luck \& Heiter 2006, Baumann et al. 2010) have found that there is no difference in lithium abundance between stars with and without planets, and they also claimed that the previously found differences were attributed to different stellar age and metallicity between stars that host planets and comparison samples. Thus it is important to perform a comparison between stars with and without planets with similar parameters (metallicity and age), and in a consistent way.

Although many studies focus on whether lithium is more depleted, or not, in dwarfs that host planets, such kind of investigations have not been taken among giants. With more and more giants with planets released by the Okayama and Xinglong planet search program, it is now possible for us to explore the lithium behavior in giants with and without planets in more detail. From Figure 5, which describes the lithium behavior against effective temperature, a remarkable feature appears: among 23 planet-hosting giants, only 8 stars have Li being detected, with a maximin of $\mathrm{A}_{\mathrm{Li}}=1.01$. However, among the 355 stars which are not known to have planets, 271 stars show Li detections. It can also be concluded from Figure 10(b-c) that differences in metallicity from the stars with and without giant planets cannot explain the diverse behavior of lithium abundances. In addition, from the H-R diagram (Figure 7), the stars with and without planets are located in the same regions, suggesting that there is no difference in terms of stellar evolution for the two sets of samples. The reason is probably due to the fact that lithium is easy to deplete in stars with planets. This effect is also unveiled among dwarfs with planets (e.g. Israelian et al. 2004, Chen et al. 2006); however, this is the first time that this aspect has been noticed for giants. Some studies(e.g. Siess \& Livio 1999, Adamów et al. 2012) found that Li-enrichment may be caused by planet accretion; however, there are no Li-rich giants in our 23 planet-hosting giants, which may suggest that such situation where the accretion of a planet leads to the formation of a Li-rich giant may be very rare. Since the number of giants with planets is limited in this sample, more targets with planets are necessary for a more detailed study.

All planet-hosting giants have vsini $<4 \mathrm{~km} \mathrm{~s}^{-1}$, which is due to the fact that giants with lower rotational velocity are more suitable for detection of planets with the radial velocity 
method, since stars with rapid rotation normally show strong stellar activity, thus there are severe variations in intrinsic RV.

\section{A large sample of lithium abundance}

To further check if the lithium abundance is a function of effective temperature and metallicity, we supplement our results with the lithium abundances from Luck07, based on the fact that the two studies are consistent in the effective temperature and the lithium abundances. There are 298 sample stars from Luck07, including $287 \mathrm{G} / \mathrm{K}$ giants and $12 \mathrm{~A} / \mathrm{F}$ giants. Luck07 used high-quality data and the spectrum synthesis method to determine the lithium abundances, which adopted by this research is based on physical parameters. Except for 96 common stars, and 15 stars without physical parameters and their corresponding lithium abundance, 187 stars are added to our sample and the final sample contains 565 giants in total. Stars with clear and undistinguished Li detections for the 378 giants are regarded as stars with $\mathrm{Li}$ detections in this section.

Non-LTE correction is applied to the 187 added stars from Luck07 using the same method described in Section 3.3. As is shown in Figure 4(b), there is no correlation between effective temperatures in the two studies for the common stars, therefore, we adopt the average value of the two works. For the metallicity in common stars, there is a slight offset, with $[\mathrm{Fe} / \mathrm{H}]_{\text {Liu2013 }}=0.04 \pm 0.01+(0.97 \pm 0.04) \times[\mathrm{Fe} / \mathrm{H}]_{\text {Luck07 }}$. There is a slight offset of non-LTE lithium abundances for stars with Li detections in both studies as well, and the correction function is $\mathrm{A}_{\mathrm{Li}-\mathrm{Liu} 2013}=0.206 \pm 0.03+(0.845 \pm 0.04) \times \mathrm{A}_{\mathrm{Li}-\mathrm{Luck07}}$. Therefore, we correct the metallicity and lithium abundance (only for stars with Li detections, and adopt the original value for stars with upper limits) from Luck07 to ours for the 187 added stars.

As Luck07 has not provided the UVW data, we calculate the kinematic parameters $\left(\mathrm{U}_{L S R}, \mathrm{~V}_{L S R}, \mathrm{~W}_{L S R}\right)$ based on the method given by Johnson \& Soderblom (1987) for all these 567 stars with radial velocities (which are already corrected to heliocentric velocities) taken from Takeda et al. (2008), Liu et al. (2010) and Luck07. The position, parallax, and proper motion are taken from Hipparcos data. A Solar motion of $(\mathrm{U}, \mathrm{V}, \mathrm{W})_{\odot}=(-10.00 \pm$ $0.36,+5.25 \pm 0.62,+7.17 \pm 0.38$ ) in $\mathrm{km} \mathrm{s}^{-1}$ (Dehnen \& Binney 1998) is adopted, where the Galactic velocity component $\mathrm{U}$ is defined to be positive towards the Galactic anticenter. The Galactic velocity components U, V, and W of stars in our program are corrected to the local standard of rest (LSR). The relative probabilities for the thick-disk-to-thin disk (TD/D)and halo-to-thick (H/TD) membership are determined using the method proposed by Bensby et al. (2003). To assign a given star to one of these groups, following the suggestion of 
Bensby et al. (2005), we adopt TD/D $\geq 2$ for thick-disk stars, and TD/D $\leq 0.6$ for thin-disk stars, and all remaining stars with a probability between these values are considered to be a member of the transition population. Among the 565 stars in the enlarged catalog, there are one star belongs to halo; 467 stars belong to the thin-disk; 43 belong to the thick-disk; and 54 are considered to be the transition population. The only halo star HD39364, with $\mathrm{H} / \mathrm{TD}=1.52$, that has an upper limit in $\mathrm{Li}$ abundance will not be included in the following comparison.

The lithium abundance as a function of metallicity and effective temperature is shown in Figure 12, separately for thin- and thick-disk stars. Black open dots, open triangles and stars represent sub-samples with Li detections, upper limits, and planets respectively for thin-disk stars, while red and blue dots, triangles and stars represent those for thick-disk stars and the transition population. In general, the lithium abundances increase towards higher effective temperature and metallicity. The enlarged sample includes 17 Li-rich giants, among which 14 are taken from Luck07, containing 9 F giants, and 5 G/K giants. HD 194937 and HD 214995 are K giants, which had been studied in Kumar et al. (2011), indicating that the enrichment may be attributed to the conversion of ${ }^{3} \mathrm{He}$ via ${ }^{7} \mathrm{Be}$ to ${ }^{7} \mathrm{Li}$ by the Cameron-Fowler mechanism. As discussed in Section 4.2, combining their positions in H-R diagram (Figure 13), the three $\mathrm{G}$ giants are also supposed to be enriched by the Cameron-Fowler mechanism. Excluding the Li-rich stars, the lithium abundance is slightly increasing with increasing metallicity and higher effective temperature for $\mathrm{G} / \mathrm{K}$ giants. Different patterns are shown for the two disks in this sample, indicating that the lithium abundances in thick-disk stars are lower than those in thin-disk in general. Meanwhile, excluding the one hot giant, there are no Li-rich and very few Li-normal stars in the thick-disk population. The different behaviors of thin-/thick-disk stars indicate that the lithium abundances in thick-disk stars are more depleted than those in thin-disk stars. The trends agree with the theoretical prediction, which expects that the thick-disk giants are older than thin-disk stars and their lithium abundances are easier to become depleted due to the higher temperature inside the giants along the RGB. The behavior of lithium abundances for giants in our sample is in agreement with the results of dwarfs and subgiants (Ramírez et al. 2012). No notable difference has been identified between stars with and without planets for thick-disk and thin-disk stars. From the metallicity distribution, the metallicity for thin-disks star are lower than those in thick-disk, supporting the theoretical prediction that thick disk stars are older. The lithium abundance, UWV value and TD/D for 565 giants are listed in Table 5, which is only available in electronic format. 


\section{Summary}

We present the lithium abundances for 378 intermediate-mass late $\mathrm{G} / \mathrm{K}$ giants by the spectrum synthesis method, among which 321 stars come from the Okayama planet search program and 57 stars from the Xinglong planet search program. The non-LTE correction is performed, resulting in a correction of 0.05-0.28 dex, with the majority around 0.18 dex. The lithium abundance as a function of metallicity, effective temperature and rotational velocity are discussed. The main results are summarized as follows:

1. Our sample includes three Li-rich giants, 36 Li-normal stars and 339 Li-depleted stars. The fraction of Li-rich stars in this sample agrees with previous works, which predict that less than $1 \%$ giants are Li-rich. Stars with normal amounts of Li are supposed to present the abundance of current interstellar medium and have experienced the expected dilution phase during the first dredge-up phase. For the 339 Li-depleted stars, the abundance of Li is depleted far more than the theoretical prediction, suggesting that the Li deficiency may have already taken place in the main sequence stage for many 1.5-5 $\mathrm{M}_{\odot}$ stars which has been carried over to the evolved $\mathrm{G} / \mathrm{K}$ giants.

2. The lithium abundance is a function of effective temperature and metallicity, but for stars within the temperature range of 4800-5100 K, there is no clear correction between lithium abundance and effective temperature.

3. The lithium abundance is not correlated with rotational velocity when it is smaller than $10 \mathrm{~km} \mathrm{~s}^{-1}$, which is consistent with the results of previous studies.

4. For giants with planets, the lithium abundance is easy to deplete. Stars with and without planets are located in the same region on the H-R diagram, suggesting that there is no difference on the stage of stellar evolution for stars with planets and those without planets. All giants harboring planets have vsini $<4 \mathrm{~km} \mathrm{~s}^{-1}$. The reason that planet-hosting giants show smaller rotational velocity is due to the fact that lower rotational velocity shows a suitable pattern to detect planets using the radial velocity method.

In Section 5, we provide a catalog of stellar parameters, lithium abundances for 565 giants, with supplementary of 187 stars from Luck07. These data are used to investigate the

lithium behavior as a function of metallicity and effective temperature, and the differences in lithium behavior for thin-/thick-disk stars. The lithium abundances slightly increase with metallicity and effective temperature in this enlarged sample of $\mathrm{G} / \mathrm{K}$ giants, and thick-disk 
stars present lower lithium abundance than thin-disk stars, reflecting different degrees of lithium depletion in these two populations.

This research is based on data collected at Okayama Astrophysical Observatory (OAO), which is operated by National Astronomical Observatory of Japan (NAOJ). We are grateful

to all of the staffs of OAO for their support during the OAO observations. This work was funded by the National Natural Science Foundation of China under grants 11173031, $11233004,11078022,11103034,11103030$ and 11390371.

\section{REFERENCES}

Adamów, M., Niedzielski, A., Villaver, E., Nowak, G., \& Wolszczan, A. 2012, ApJ, 754, L15

Baumann, P., Ramirez, I., Meléndez, J. et al. 2010, A\&A, 519, 87

Barklem, P. S., O’Mara, B. J., \& Ross, J. E. 1998, MNRAS, 296, 1057

Bensby, T., Feltzing, S., \& Lundström, I. 2003, A\&A, 410, 527

Bensby, T., Feltzing, S., Lundström, I., \& Ilyin, I. 2005, A\&A, 433, 185

Boesgaard, A. M., \& Tripicco, M. J. 1986, ApJ, 303, 724

Brown, J.A., Sneden, C., Lambert, D.L., \& Dutchover, E. 1989, ApJS, 71, 293

Cameron A.G.W., \& Fowler W.A. 1971, ApJ, 164, 111

Charbonnel, C., \& Do Nascimento, J.D.Jr. 1998, A\&A, 336, 915

Charbonnel, C., \& Balachandran 2000, A\&A, 359, 563

Chen, Y. Q., \& Zhao, G., 2006, AJ, 131, 1816

Dehnen, W., \& Binney, J. J., 1998, MNRAS, 298, 387

de Laverny, P., do Nascimento, J. D. Jr., Lèbre A., \& De Medeiros, J. R. 2003, A\&A, 410, 937

De Medeiros, J. R., do Nascimento, J. D., Jr. \& Mayor, M. 1997, A\&A, 317, 701

De Medeiros, J. R., do Nascimento, J. D., Jr., Sankarankutty. S., Costa, J. M., \& Maia, M. R. G., 2000, A\&A, 363, 239 
Fuhrmann, K., Pfeiffer, M., Frank, C., Reetz, J., \& Gehren T. 1997, A\&A, 323, 909

Ghezzi, L, Cunha, K., Smith, V. V., \& Reza, R. de la. 2010, ApJ, 724, 154

Gonzalez, O. A., Zoccali, M., \& Monaco, L. et al. 2009, A\&A, 508, 289

Iben, I. J. 1965, ApJ, 142, 1447

Iben, I.J. 1966, ApJ, 143, 483

Iben, I.J. 1967a, ApJ, 147, 624

Iben, I.J. 1967b, ApJ, 142, 650

Israelian, G., Santos, N., Mayor, M., \& Rebolo, R. 2004, A\&A, 414, 601

Israelian, G., Delgado, M.E., Santos, N.C. et al. 2009, Nature, 462, 189

Johnson, D.R.H., \& Soderblom, D.R. 1987, AJ, 93, 864

Kumar, Y.B., Reddy, B.E., \& Lambert, D. 2011, ApJ, 730, L12

Lèbre, A., De Laverny, P., De Medeiros, J. R., Charbonnel, C., \& Da Sila, L. 1999, A\&A, 345,936

Lèbre, A., De Laverny, P., Do Nascimento J. D., \& De Medeiros J. R. 2006, A\&A, 450, 1173

Lind, K., Asplund, M., \& Barklem, P.S. 2009, A\&A, 503, 541

Liu, Y. J., Sato, B., Zhao, G. \& Ando H., et al. 2008, ApJ, 672, 553

Liu, Y., Sato, B., Takeda, Y., Ando, H., \& Zhao, G. 2010, PASJ, 62, 1071

Luck, R. E., \& Wepfer, G. G. 1995, AJ, 110, 2425

Luck, R. E., \& Heiter, U. 2006, AJ, 131, 3069

Luck, R.E., \& Heiter, U. 2007, AJ, 133, 2464

Mallik, S. V., Parthasarathy, M., \& Pati, A. K. 2003, A\&A, 409, 251

Randich, S., Pasquini, L., \& Pallavicini, R. 2000, A\&A, 356, 25

Ramírez, I., Fish, J. R., Lambert, D. L. \& Allende Prieto, C. 2012, ApJ, 756, 671

Reddy, B. E., Lambert, D. L., \& Allende Prieto, C. 2006, MNRAS, 367, 1329 
Reetz, J. K. 1993, unpublished

Sato, B., Ando, H., Kambe, E. et al., 2003, APJ, 597, L157

Siess, L., \& Livio, M., 1999, MNRAS, 308, 1133

Takeda, Y., \& Kawanomoto, S. 2005, PASJ, 57, 45

Takeda, Y., Sato, B., \& Murata, D. 2008, PASJ, 60, 781

Takeda, Y., Honda, S., Kawanomoto, S., Ando, H., \& Sakurai, T. 2010, A\&A, 515, 93

Takeda, Y., Kang, D., Han, I., et al., 2012, PASJ, 64, 38

Wallerstein, G., Bohm-Vitense, E., Vanture, A. D., \& Gonzalez, G. 1994, AJ, 107, 2211

Wang, L., Liu, Y.J., Zhao, G., \& Sato, B. 2011, PASJ, 63, 1035

Yi, S.K., Kim, Y.-C., \& Demarque, P. 2003, ApJS, 144, 259 


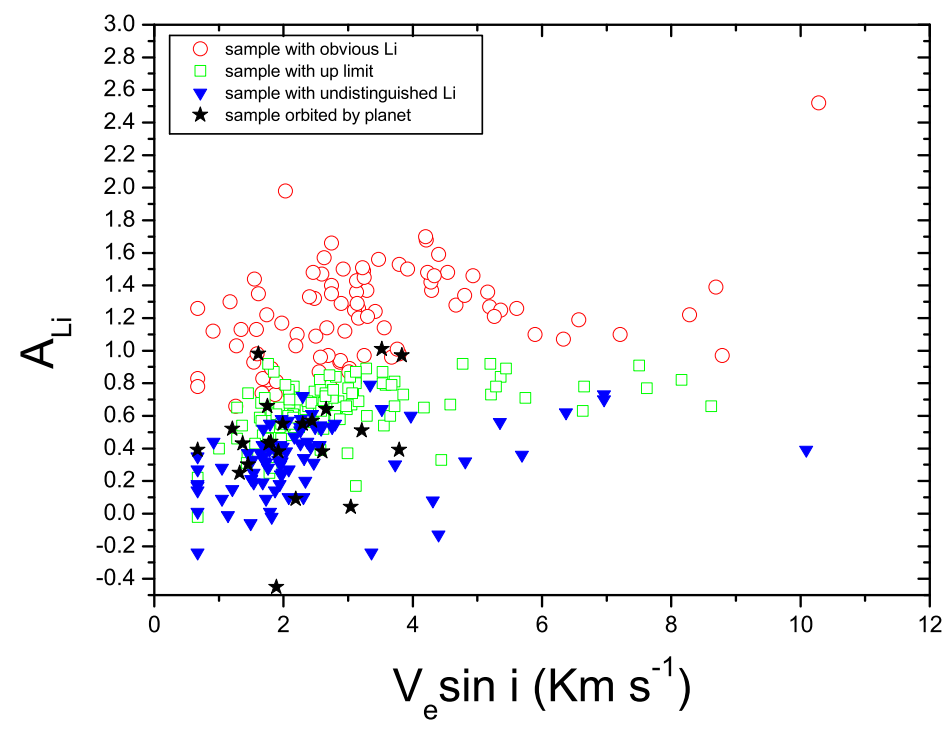

Fig. 10.- Lithium abundance versus rotational velocity. The symbols are the same as in Figure2. 

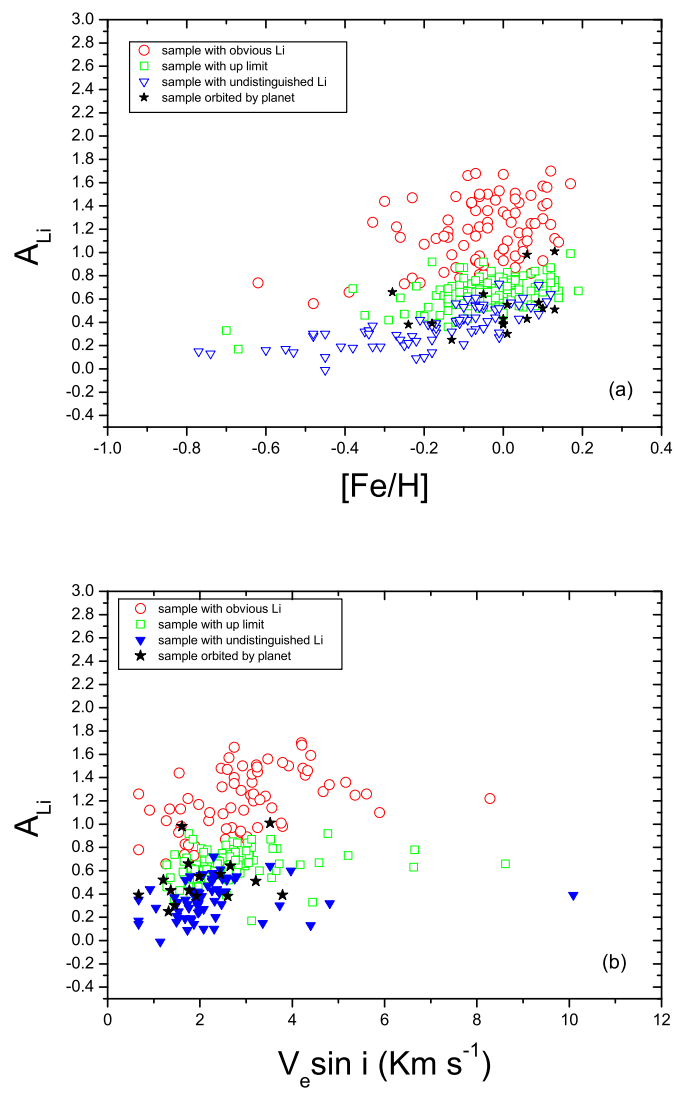

Fig. 11. - Lithium abundance against metallicity, and rotational velocity when $4800 \leq \mathrm{T}_{\text {eff }}$ $\leq 5100 \mathrm{~K}$. The symbols are the same as in Figure2. 

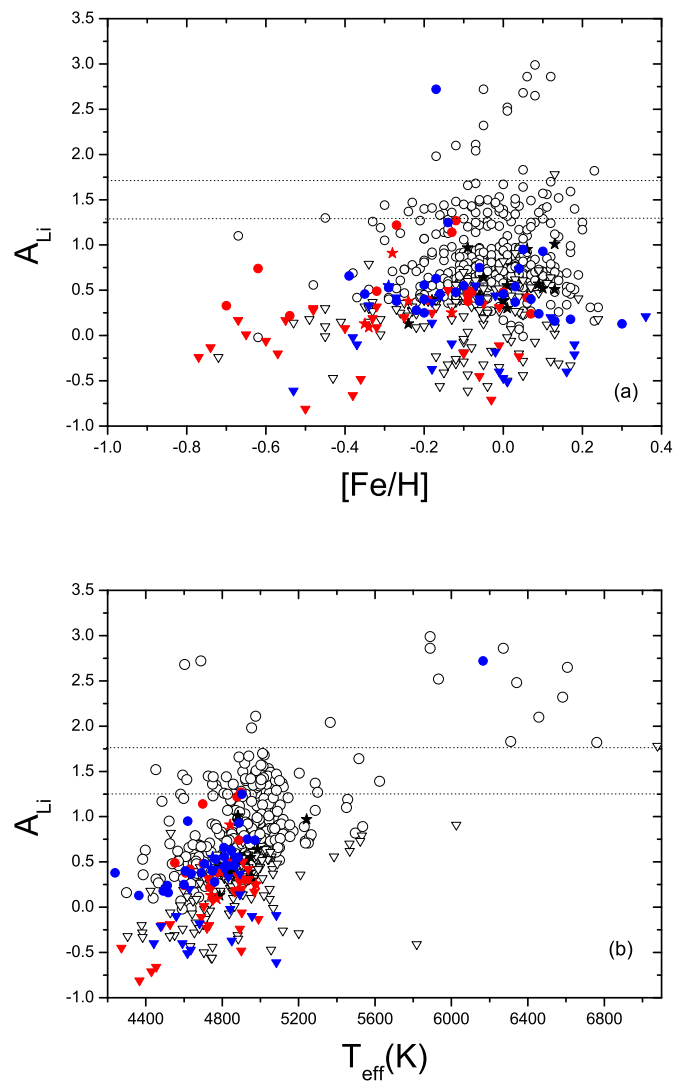

Fig. 12.- Lithium abundance against metallicity and effective temperature. The black open dots, open triangles and stars represent sub-sample with Li-detections, upper limits and planets for thin-disk, while red and blue dots, triangle and stars represent those for thick-disk stars and transition population. 


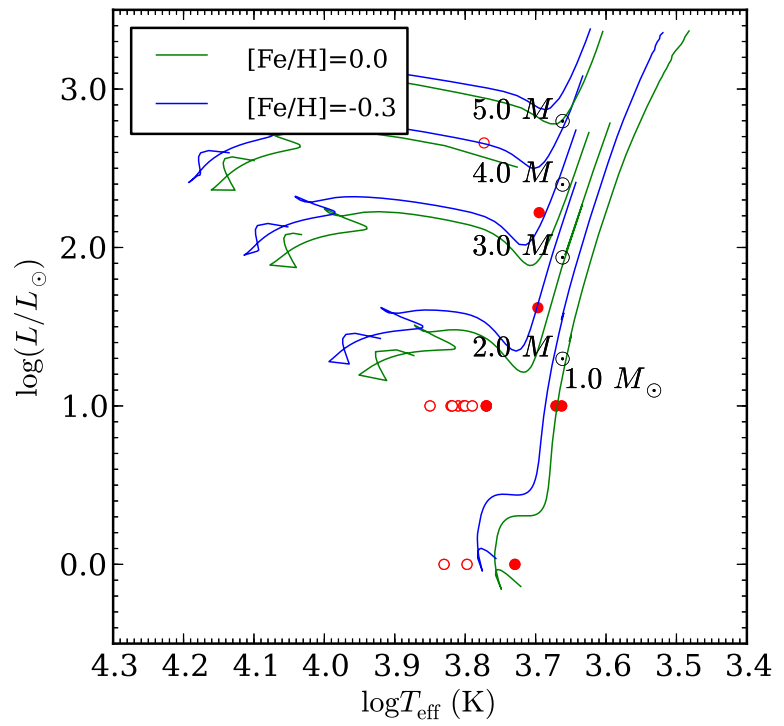

Fig. 13. - Lithium abundance for 17 Li-rich stars in H-R diagram. Open dots and filled dots represent giants with a spectral type of $\mathrm{F}$ and $\mathrm{G} / \mathrm{K}$. 